\title{
The Visual Inspection of Key Pad Parts Using a Fuzzy Binarization Algorithm
}

\author{
Young-Baek Kim, Hong-Chang Lee and Sang-Yong Rhee ${ }^{+}$ \\ Division of Computer Science and Engineering, Kyungnam University \\ 449 Woreong-dong, Changwon, Gyeongnam, Korea
}

\begin{abstract}
The detection of defective parts in a factory is usually performed by the human eye. Therefore, heavy manpower is in demand for minor enterprises. An image processing system is desired to solve this drawback. However, due to the variety of the products characteristics, an general algorithm is needed that can adapt to these characteristics. Therefore, in this paper, the key pad parts' characteristics which need to be dealt with are analyzed in order to embody the image processing algorithm that is suggested. The experimental results show the probability of detecting a defective part is $95 \%$ with a detection time of 0.203 seconds, on the average.
\end{abstract}

Keywords : Visual inspection system, Fuzzy binarization, mobile phone parts' feature

\section{Introduction}

Detecting the inferior parts that are produced in a small enterprise factory is usually handled by a trained labor force. These labor forces are developed with a long period investment and since this process involves human perception it has a low productivity, because the extent of detection is dependant on the expert's abilities. This system also needs a large labor force so the increase of labor costs becomes a problem as well. Therefore an automatic detection system is needed that easily detects inferior produced parts as well being easy to operate for all people, not just experts. Most of these automatic detecting system work in place of human eyes. So these systems use a camera that does same role as the eyes and by doing this many image processing methods are used in which images are inputted and handled.

Through this process, one can strengthen the competitiveness of minor enterprises, increasing their productivity and reducing labor costs.

But the standards of the superiority and the inferiority of various products, including the key pad parts that are dealt with in this paper, are quite different from each other so applying one certain algorithm to all products isn't easy. Furthermore, the one algorithm will be outperformed by an algorithm which considers the individual characteristics of each product in speed and accuracy. Because of this, the key pad parts characteristics are exactly analyzed and applied to an image processing algorithm that bring these characters into sharp relief and so detects the inferior key pad parts.

Manuscript received Nov. 29, 2010; revised Jul. 6, 2011; accepted Jul. 6, 2011.

${ }^{+}$Corresponding author

This work was supported by Kyungnam University Foundation Grant.in 2010.

\section{Analyzing the Superior Characteristics of Key Pad Parts}

The parts that are used for this study are an attachment for a mobile phone. As seen in Fig. 2.1, it is made of PVC (polyvinylchloride) and it has a combination of a rectangular base with small PVC pieces on the top.

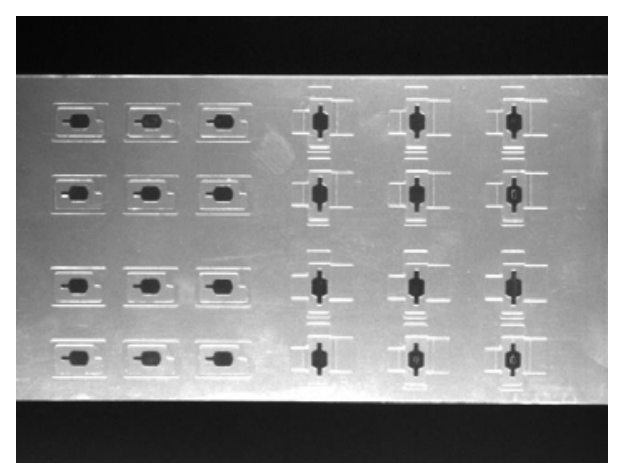

Fig. 2.1 An image of the key pad parts

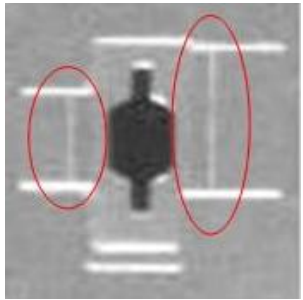

(a) Good

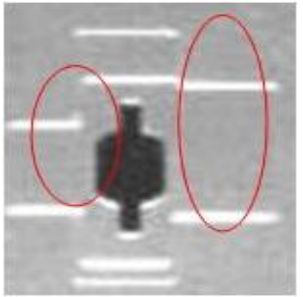

(b) Inferior
Fig. 2.2 The parts of key pad

Sometimes during the attachment of the small parts to the base an inferior process causes a weak attachment between the small part and the base.

In image 2.2, (a) is a good part where the small piece is attached normally to the base and (b) is an inferior part in which the small piece has come off from the base. 
An accurate judgment is required from the obtained image in order to check that the small part is stuck to the base.

The standard that determines a good part from bad is the existence of a white vertical line, as shown in Fig. 2.2. This line only exists in a good part during the process of pasting a thin coating sheet to prevent the small part from falling apart or being shifted by an external force after the attachment of the small part onto the top of the base. When observing this process, the section where the small part is attached has a gap in the height as well as in the length of the part. Because of this, the coating sheet cannot stick flat with the basic pad and forms an empty space the same length as the height gap. When this empty gap is reattached to the basic part, the region turns a little opaque, which is shown by white vertical lines like in Fig 2.2(a). On the other hand, the inferior part has no small piece on the top of the base, so the coating sheet is stuck flat with no empty space so the opaque vertical lines do not appear. Therefore, if the system can correctly judge the existence of the white vertical lines that only exist in the good part, the inferior detection can proceed with accuracy. But these lines are quite vague and require a minute observation even for a human eye. Accordingly, judging the existence of the vertical white line divides into two sections which are "quite exists" and "does not exist" but the standard of this "quite" is vague in accordance the lighting conditions and the perspectives of the inspector so an inferior detecting algorithm is suggested in order to solve the vagueness by using the fuzzy binarization method.

\section{The Inferior Detection Method}

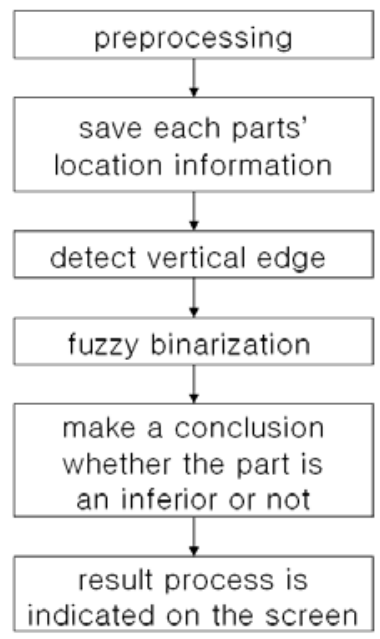

Fig. 3.1 The inferior detection process

In this study the discrimination of inferior parts is judged by a detected vertical edge which only exists in a good product. In the first step, the binarization is processed in order to get an accurate location of the parts. The second step is to save each parts' location information from the whole image. Next, in the third step, we use a detected vertical edge and fuzzy binarization which only shows the standard features of the good parts from the original image. The fourth step is to compare a parts' location information from step 2 and the standard feature from step 3 to made the conclusion of whether the part is inferior or not. In last step the results of the process are indicated on a screen.

\subsection{Detecting the special features of a good part.}

When the mobile-phone key pad parts are pictured through a vision camera we get a different image dependant on the condition of the product, either good or bad.

The good ones have both left and right white stripes in their images as seen in Fig. 2.2(a). However, the inferior parts don't have them. To figure out the feature of the good in the acquired image, we first detect vertical edges. The Sobel vertical mask is used, which is one kind of a first order differential operator in order to detect the vertical edges.

Next, we binarized the detected vertical edge image. We modified Yoon's fuzzy binarization[6] for this research. The general method of binarization is presented as equation (1). It substitutes a threshold for the average of the brightest pixel and the darkest pixel in the original image. Setting a standard as the threshold, we can divide them into two groups which are 0 for the bigger pixels and 1 for the smaller pixels[5].

$$
T=\frac{I_{\max }+I_{\min }}{2}
$$

In (1), $T$ refers to a threshold, $I_{\max }$ to the pixel of maximum brightness and $I_{\min }$ to the pixel of minimum brightness.

However it is hard to get a good results by using the threshold obtained from this method. That is why there is fuzziness in selecting the threshold for detecting the existence of the feature points.

To solve this fuzziness, a fuzzy binarization method was suggested. However the existing method[5] used the entire image data, so it was not suitable for an edge detected image whose feature points were already filtered to some extent. In short, if the pixel value was 0 in edge detected image, it meant there was no information at all. Therefore in this paper, the remaining data excluding the pixel value 0 was applied to process the fuzzy binarization.

Each $X_{i}^{r}, X_{i}^{g}$ and $X_{i}^{b}$ are defined as a result of the inputted image's RGB values and the calculation of the medium brightness $X_{m}$ uses this value as the following:

$$
\begin{aligned}
& X_{m}=X_{m}+\frac{X_{i}^{r}+X_{i}^{g}+X_{i}^{b}}{3}, \quad \text { if } \frac{X_{i}^{r}+X_{i}^{g}+X_{i}^{b}}{3}>0 \\
& =\frac{X_{m}}{\text { count }}, \quad \text { otherwise }
\end{aligned}
$$

Then we calculated the distance value of the dark range Dmin and the distance value of the bright range Dmax by using $X_{m}$. 


$$
\begin{aligned}
& D_{\text {max }}=\left|X_{h}-X_{m}\right| \\
& D_{\text {mun }}=\left|X_{m}-X_{l}\right|
\end{aligned}
$$

In this point, $X_{l}$ is the value of the darkest pixel and $X_{h}$ is the value of the brightest pixel in the input image.

We also calculated the maximum brightness value Imax and the minimum brightness value Imin by applying Dmin and Dmax to following rules:

$$
\begin{aligned}
X_{m} & =255-X_{m} & & \text { if } X_{m}>128 \\
& =X_{m} & & \text { otherwise } \\
I_{\min } & =X_{m}-X_{m} & & \text { if } D_{\min }>X_{m} \\
& =X_{m}-D_{\min } & & \text { otherwise } \\
I_{\max } & =X_{m}+X_{m} & & \text { if } D_{\max }>X_{m} \\
& =X_{m}+D_{\max } & & \text { otherwise }
\end{aligned}
$$

The brightest value Imax and the least bright value Imin were applied to get a triangle type membership function.

Therefore the of triangle type membership function, has an interval. In the membership function, we calculated the medium bright value Imid to make the membership 1, as follows:

$$
I_{\text {mid }}=\frac{I_{\max }+I_{\min }}{2}
$$

Also the membership of section |Imin, Imax $\mid$ can be calculated as in (6). We applied cut membership ( . x) which was gotten before in the membership function to make the image binarization. At this point, we set the value as 0.5 . According to this function to make a binary image, when the membership is higher than 0.5 , we set the value of image's pixel as 255 , and when it is lower the value of image's pixel as 0 .

$$
\begin{array}{rlr}
\mu(x) & =0, & \text { if } X_{m} \leq I_{\min } \text { or } X_{m} \geq I_{\max }, \\
& =\frac{\left(I_{\max }-X_{m}\right)}{\left(I_{\max }-I_{\text {mid }}\right)} & \text { if } X_{m}>I_{\text {mid }}, \\
& =\frac{\left(X_{m}-I_{\text {min }}\right)}{\left(I_{\text {mid }}-I_{\text {min }}\right)} & \text { if } X_{m}<I_{\text {mid }}, \\
& =1 & \text { if } X_{m}=I_{\text {mid }},
\end{array}
$$

Fig. 3.2 (a) shows an image of the detected vertical edges and (b) presents an image of the applied fuzzy binarization to image (a).

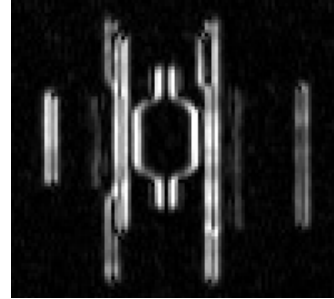

(a) Vertical Edge Image

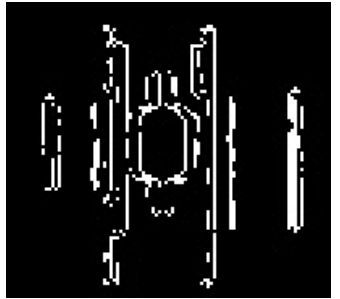

(b) Fuzzy Binarization

\subsection{Judging the inferior part by using the location data and} the features of the good part.

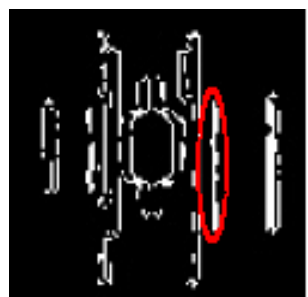

(a) Good

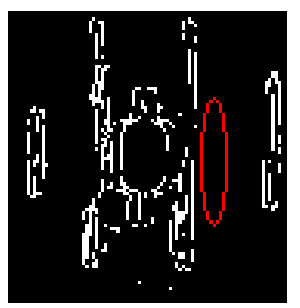

(b) Inferior
Fig. 3.3 The vertical edges detected from the original image.

Fig. 3.3 (a) shows the vertical edges of the good part and (b) is a result of detecting an inferior part's vertical edges. The vertical edges of (a) have appeared clearly at a pixel thickness. The vertical edge on the left side can be classified into two different kinds, but this doesn't affect the detected result because it detects the pixel equal to the numerical value of the part's height. We use this resultant image to find inferiors.

The good image has the features of a consistency of height and location. Therefore each part's location data is collected

through the second step and by the third step the features of the good one have been detected. Now the decision of whether the parts are good or not in the third process resultant image by using the parts location data.

Searching for the detected edge starts from the maximum width of each part moving toward the right side and the minimum and the maximum - the upper and the lower - height. If the edge was detected, it is considered to be a good one and if not, an inferior one. Fig. 3.4 shows the parts that are considered to be inferior checked into boxes.

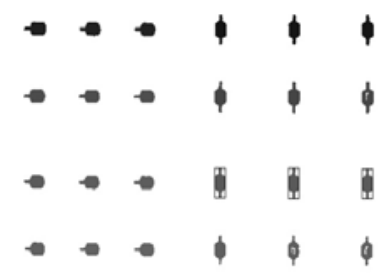

Fig. 3.4 Detecting the inferior parts 


\section{The Experimental Results and analysis}

For analyzing the efficiency of the algorithm that is suggested in this study, the algorithm process, which was composed of an image obtaining device and an Intel Core 2 Duo Processor and 3GB memory PC using the Intel OpenCV library of and Visual $\mathrm{C}++6.0$, was reviewed.

The image obtaining device used image 3.1 for its composition. A 1/4.5 inch Bayer Mosaic CMOS sensor was installed and a hyvision company's vision camera HVR_2030R was fixed in the middle upper portion with a $640 \times 480$ resolution and a USB 2.0 interface. After this, a black colored table was put on the floor to increase the contrast of the image and a white bar-type LED lighting fixture was installed on each side so the light could bat on the parts evenly. To determine the best lightening condition, we tested lighting angles of $0^{\circ}$, $45^{\circ}$ and $90^{\circ}$. The results of the $0^{\circ}$ test were similar to that of the $45^{\circ}$ test and the two results were better than the $90^{\circ}$ test. In the $90^{\circ}$ case, the vertical features were less shown. The features were better shown by parallel lightening. Therefore our experiments were tested after fixing the light to the $0^{\circ}$ angle.

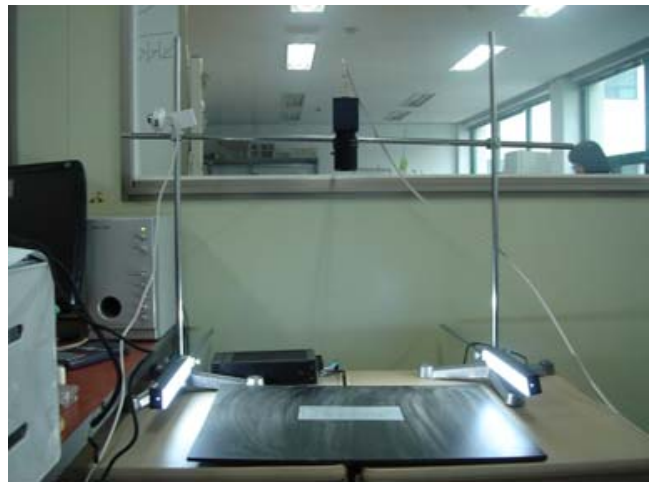

Fig 4.1 The image acquisition device.

Estimating the efficiency proceeded by using a hundred 640x480 sized key pad parts images. These 100 images were composed of 96 superior images and 4 inferior images.

In this paper in order to detect the characteristics of the superior parts in the obtained image a vertical edge was detected and processed by binarization on the detected edge image. Also applied were the edge image binarization methods to the fuzzy binarization method and compared to other binarization methods.

Image 4.2 is a picture of the detecting edge using fuzzy and other binarization methods. (a) in image 4.2 is the suggested fuzzy binarization method. (b) is Otsu[7] method, a representative binarization method, that decides threshold value automatically and (c) is an image of a binarization method that manually determines the threshold value on several images and puts the average threshold value as a representative threshold value. By observing image 4.2, the fuzzy binarization showed most clearly the characteristics of the superior part, while the Otsu method did not show the characteristics of the superior key pad parts at all. The fuzzy binarization method fuzzified the edge quantity that the character of superior key pad parts have. On the other hand Otsu binarization method had a standard assorting class by simple intensity of the edge which can not detect the vagueness that the superior characteristics have. Also the binarization method using the representative threshold value has a worse result than the fuzzy binarization method because it is hard to apply a vagueness caused by a change in the environment.

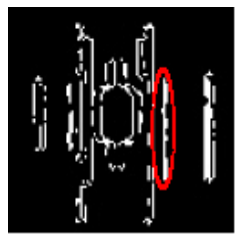

(a) Fuzzy binarization method

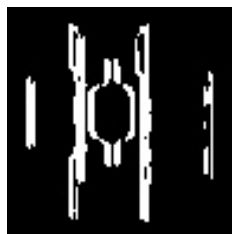

(b) Otsu binarization method

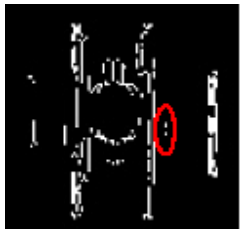

(c) epresentative threshold value binarization method
Fig. 4.2 The results of the fuzzy binarization method and other binarization methods

Fig. 4.3 is a graph that shows the comparison of the superior's characteristics detecting efficiency between the fuzzy binarization method and the other binarization methods. The $\mathrm{Y}$ axle is the detected amount of pixels that correspond to the superior's key pad parts characteristics.

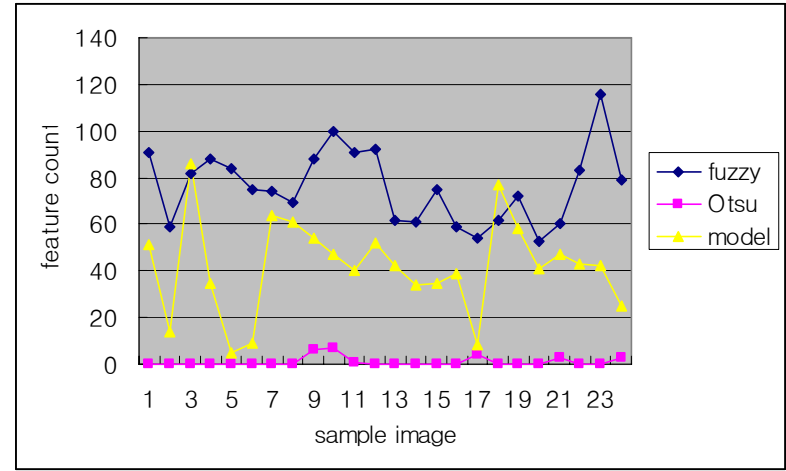

Fig 4.3 The efficiency comparison between the fuzzy and the other binarization methods.

The results of Fig. 4.3 indicate that the Otsu binarization method could not detect the superiors' character at all because Otsu binarization method simply applied the threshold value that analyzed the frequency histogram. In fact, the difference between the edge characteristic and the basic part that does not have the edge is so slight that using a Otsu binarization method, which divides the classes into parts which have a strong edge and parts which do not, will find it hard to distinguish the two classes by just applying the frequency histogram, because in this method the characteristics are closer to the parts that do not have an edge.

But the fuzzy binarization method showed a very high 
detecting percentage. This is because this method uses a membership function that gives weight on information of the edge in point. This membership function made the method detect a slight difference between the basic part and the superior characteristics. The representative threshold value binarization method has an uneven and a smaller detecting percentage than the fuzzy. This indicates that even though the threshold value had been decided manually, there is a limit in detecting the slight difference of the basic and the edge characteristics and by using an average threshold value the error worked a big role. As the experimental images are not taken in the same time, changes in circumstances such as lighting cannot be ignored. But in the representative threshold value binarization method the vagueness according to the change of circumstance was not considered so the error was amplified. The fuzzy binarization method applied the revised membership function which fits for each image's information so that it gains a more stable and accurate result.

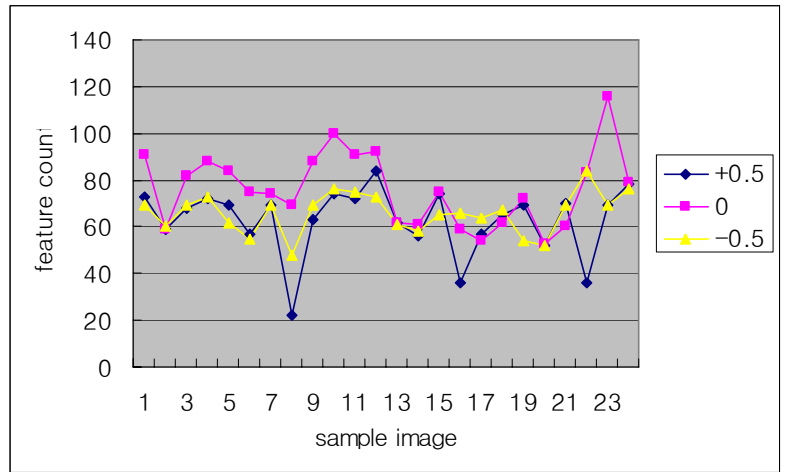

(a) The fuzzy binarization method

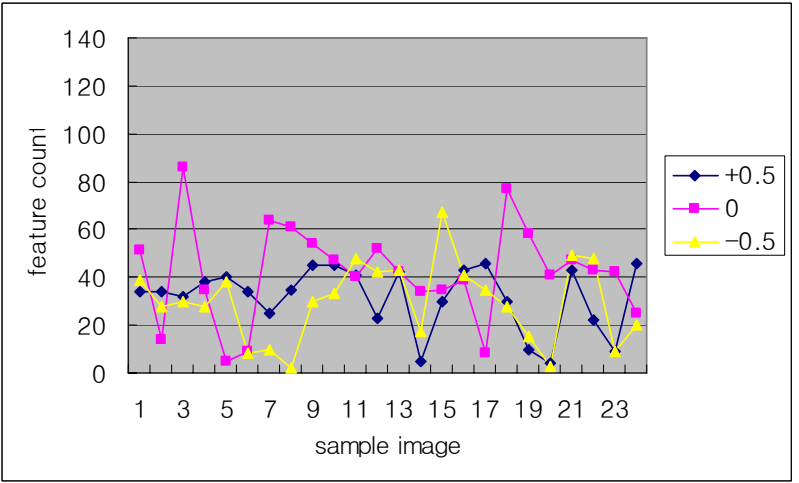

(b) The representative threshold value binarization method

Fig. 4.4 The comparison of the binarization method dependant on differences in exposure

The next thing tested indicates difference in the result between each binarization method when the exposure was changed. Of course the photometry ability of a camera differs in each model so under our circumstances the result had only \pm 0.5 step of errors so these test parameters were used. Fig. 4.4 is a graph that shows the efficiency comparison change according to various exposures. Fig. 4.4 (a) indicates the fuzzy binarization method and (b) shows the representative threshold value binarization method. The results appear to be very different from each other. Graph (a) shows a comparatively even result from exposure change but graph (b) has an uneven result and sometimes did not detect the superior edge characteristics at all. In the case of the fuzzy binarization method, it has an even result because it has a membership function that adapts itself to the brightness of the image. On the other hand, the representative threshold value binarization method applied the first fixed threshold value, so the result turned out totally different according to the brightness of the image.

The total processing time it takes to detect an inferior part after obtaining the image is shown in Table 1, which divided time into seconds using the different binarization methods.

Table 1. The Inferior Detection Time

\begin{tabular}{|c|c|c|c|}
\hline & Fuzzy & Otsu & model \\
\hline Time(sec) & 0.203 & 0.191 & 0.188 \\
\hline
\end{tabular}

The data that is shown in Table 1 is an average value obtained by repeating the test process 100 times

As an experimental result, the fuzzy binarization method took $0.015 \mathrm{sec}$ more than the representative threshold value method due to the inclusion of the operation that decided the threshold value while processing the detection, unlike the representative method which already has a threshold value obtained at the start. However this overhead operation is needed for an automatic detecting device that is strong for changes of circumstances and has a high accuracy.

Table 2. The Experimetal Results

\begin{tabular}{|c|c|c|c|c|}
\hline & Correct Accept & Correct Reject & FRR & FAR \\
\hline Fuzzy & $91 / 96$ & $4 / 4$ & $5 / 96$ & $0 / 4$ \\
\hline Otsu & $8 / 96$ & $4 / 4$ & $88 / 96$ & $0 / 4$ \\
\hline Model & $87 / 96$ & $4 / 4$ & $9 / 96$ & $0 / 4$ \\
\hline
\end{tabular}

Table 2 shows the results of the detection of the inferior key pad parts using each different binarization method. A total of a hundred key pads were tested which included 4 inferior key pads. The experimental results show that the fuzzy binarization method has the highest detection rate of the three different methods by finding 91 superior samples out of a total of 96 . The 5 that were not detected were because during the process of laying the key pad parts on the black board the parts did not adhere closely. In this case, when the part bat the lighting, the reflexibility of the light changed due to a specific character of the parts' material so that the superior characteristics did not show in the image. Therefore, when excluding these cases, the percentage of detecting is $100 \%$ which shows an excellent efficiency. A case that judged an inferior as a superior did not happened in all three binarization methods. The reason was that the algorithms that are suggested in this study handled the parts as an inferior when the analyzed superior characteristic is not 
found. In an automatic detection system adopting these kinds of algorithms work best for reducing a financial loss because judging an inferior as a superior can bring a much bigger financial loss than the opposite.

\section{Conclusions}

In this study, we suggested an inferior part detection method using specified features of a mobile-phone parts' image acquired by a vision camera. Through fuzzy binarization, the location data of parts was investigated and the vertical edge was detected. Whether each part is superior or inferior can be detected by using the features found only in the superior. The experiment was held in a fixed environment, with a detection ratio of $95 \%$ and an average detection time of 0.203 seconds.

\section{References}

[1] Sun-Kyoo Hwang, Image Processing by Visual C++, Hanbit Media, pp 34 35, 2007. 11.

[2] Wn-Goo Kim, Myong-Ho Kang, Jong-Kug An, In-BoJeong, and Sung-Hyn Han, "Design of Intelligent Vision System for Automatic Inspection of Parts," Proceedings of the Korea society for Manufacturing Process Engineers, pp. 284 288, 2006.11.

[3] S. J. Kim, S. C. Lee, K. E. Yang, "Development of Automotive HID lamp Inspection Machine using Vision System," Proceedings of the Korea society for precision engineering, pp.517 518, 2008.11.

[4] Ju-Young Yoon, Young-Choon Lee, Doo-Yeol Pang, and Seong-Cheol Lee, "Surface Inspection System of Bearing Inner/Outer Race using Machine Vision," Journal of the Korea society for precision engineering, pp.309 310, 2006.5.

[5] Hyung-Keun Yun, Ji-hoon Lee, Kwang-Baek Kim, "A Study on Fuzzy Bionarization Method," Korea Intelligent Information Systems Society, vol. 2, no. 11, pp. 510 513, 2002.

[6] Doo-Eok Cheon, Sung-Ho Yoon, Kwang-Baek Kim, "A Study on Image Recognition using Enhanced ART1 Algorithm," Journal of the korea society of computer and information, vol. 3, no. 3, pp.15 20, 1998.
[7] N. Otsu, "A threshold selection method from gray-level histograms," IEEE Trans System, Man and Cybernetics, vol. 9, no. 1, pp. 62-66, 1979.

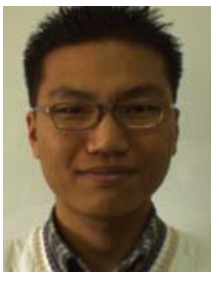

Young-Baek Kim received the B.S. and M.S. degrees in computer science and engineering from Kyungnam University, Gyungnam Masan, Korea in 2005 and 2007, respectively. He is currently in the Ph.D. course. His current research interests include image processing, context awareness and augmented reality.

E-mail : baroaleum@gmail.com

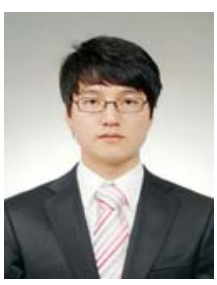

Hong-Chang Lee received the B.S. degree in in computer science and engineering from Kyungnam University, Gyungnam Masan, Korea in 2009. He is currently in the M.S. course. His current research interests include image processing, computer vision and motion analysis

E-mail : square1004@hanmail.net

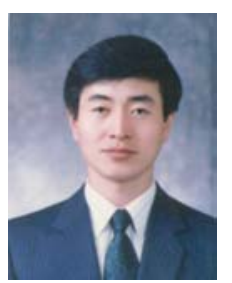

Sang-Yong Rhee received the B.S. and M.S. degrees in industrial engineering, Korea University, Seoul, Korea in 1982 and 1984, respectively and the Ph.D. degree in industrial engineering, Postech, Gyeongbuk Pohang, Korea in 1992. He is currently a Full Professor in the Division of Computer Science and Engineering, Kyungnam University, Korea. His current research interests include computer vision, neuro-fuzzy system, intelligent robot and augmented reality.

E-mail : syrhree@kyungnam.ac.kr 